Supporting information

\title{
Solid-State Fluorescent Carbon Dots for Fluorimetric Sensing of $\mathbf{H g}^{\mathbf{2 +}}$
}

Chandra Mohan Singaravelu*, Xavier Deschanels, Cyrielle Rey and Jérémy Causse*

ICSM, University of Montpellier, CEA, CNRS, ENSCM, 30207 Marcoule,France,Email. chemcm@gmail.com, jeremy.causse@cea.fr

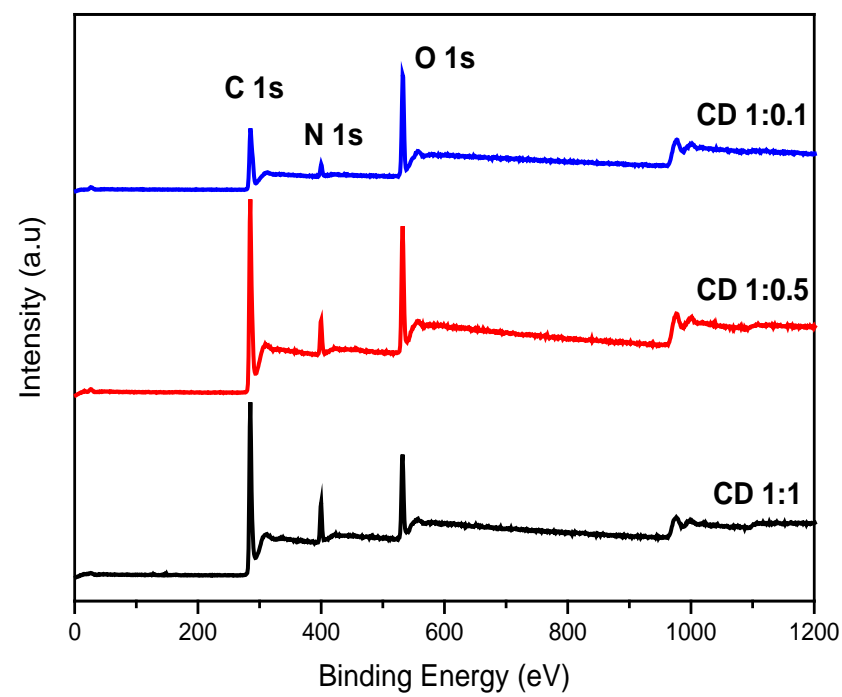

Figure S1. XPS survey spectrum of CDs (CD1:1, CD1:0.5 and CD1:0.1).

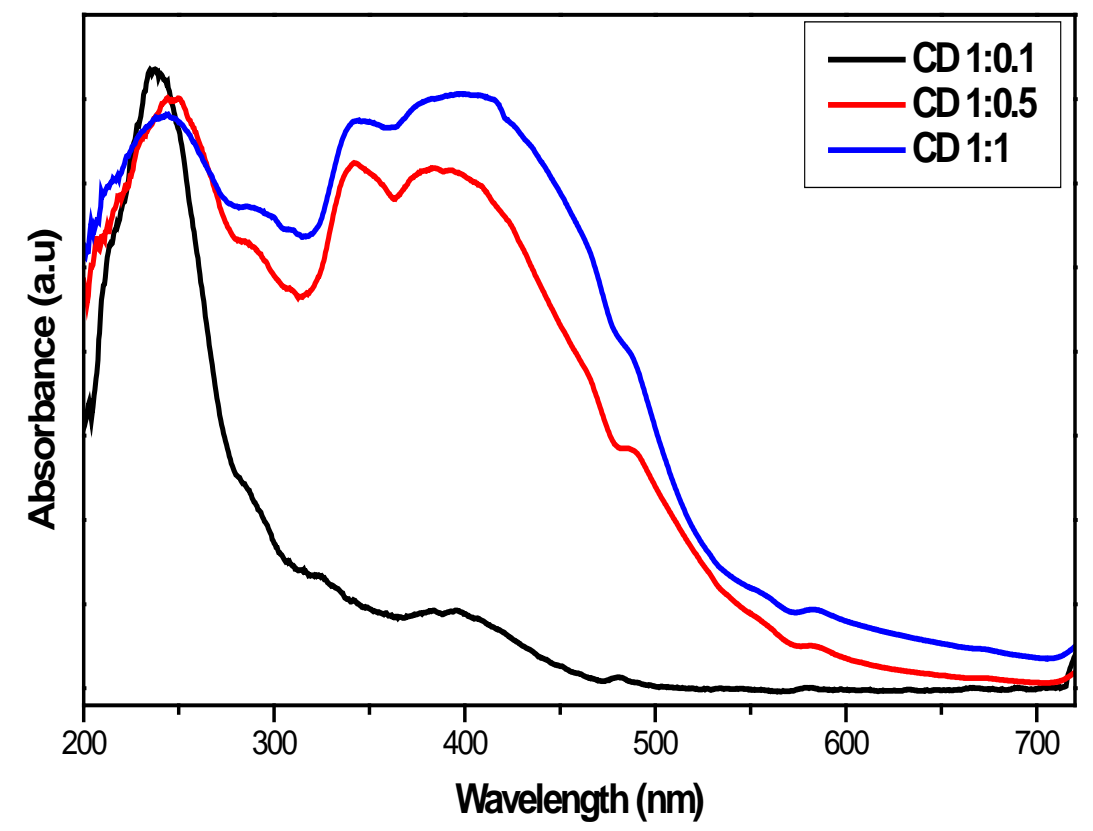

Figure S2. DRS UV-Visible absorption spectra of solid-state CDs (CD1:1, CD1:0.5 and CD1:0.1). 

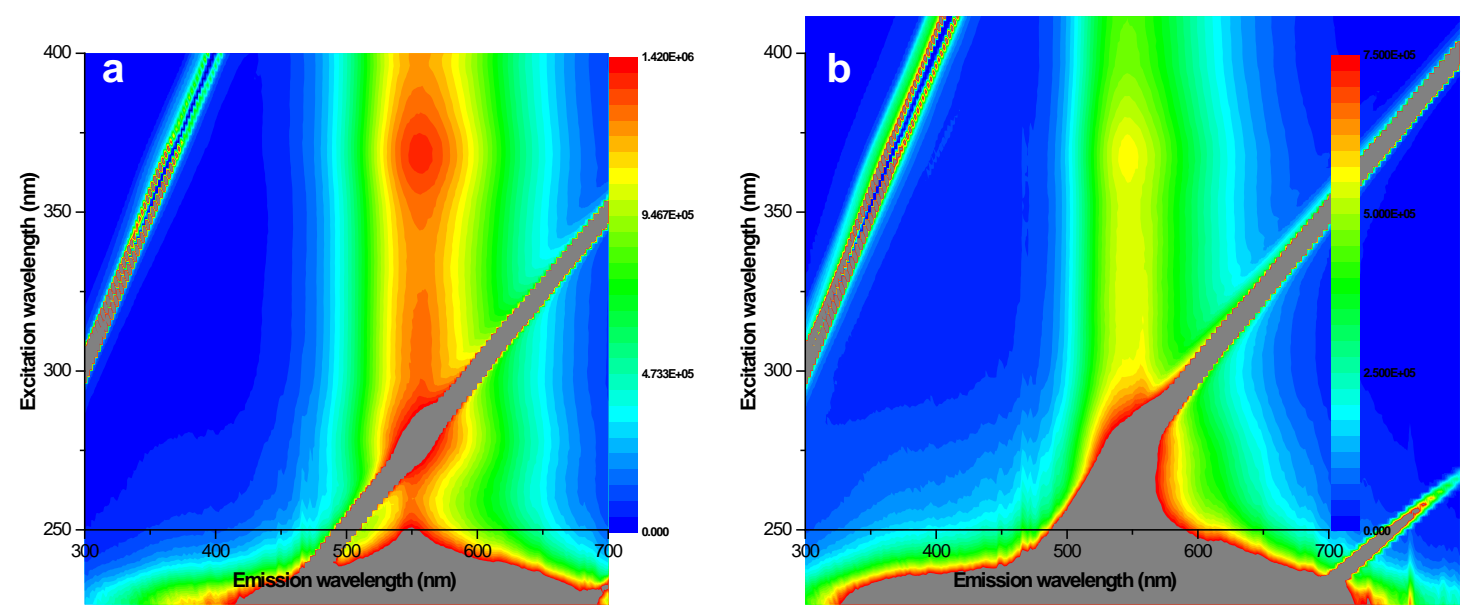

Figure S3. 2D fluorescence topographical map of solid state CDs CD1:05(a), CD1:1(b).
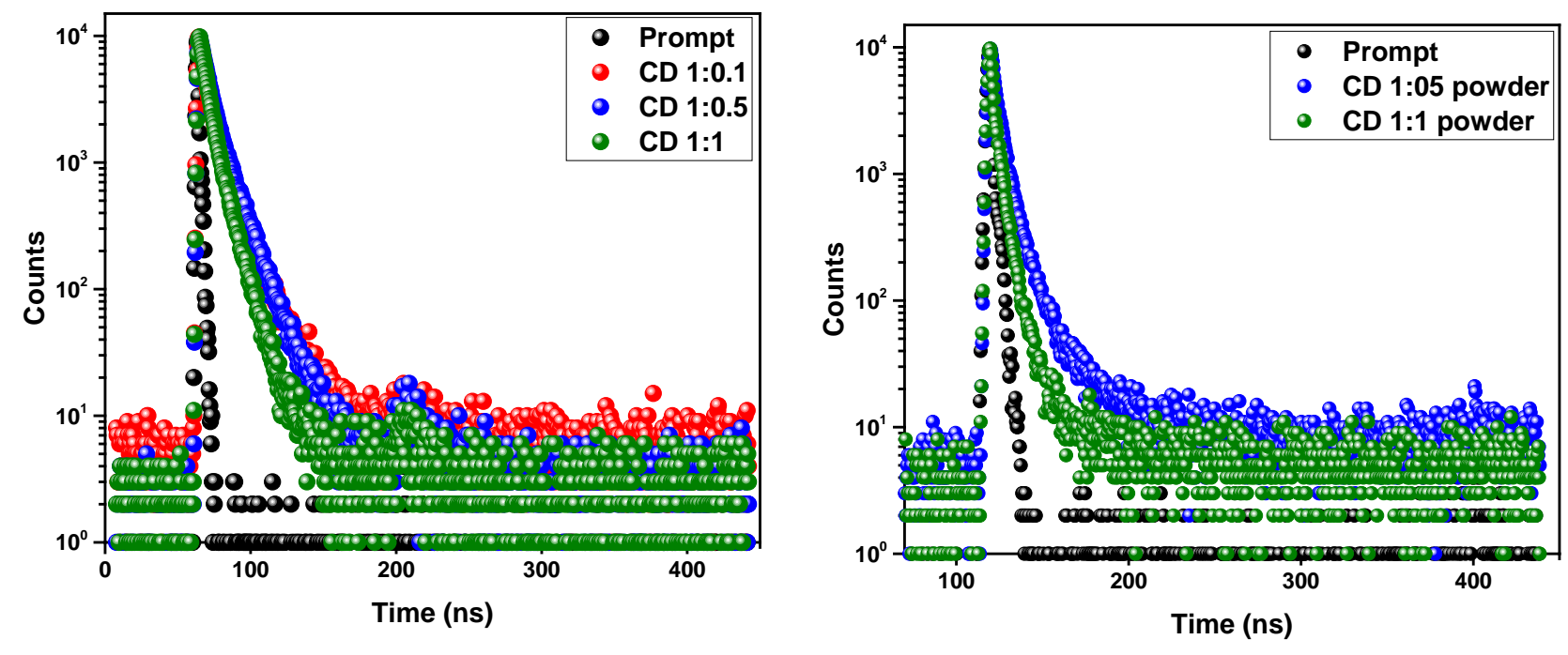

Figure S4. Photoluminescence decay profile of CDs in solution (a) and powder (b) monitored at respective emission maxima of CDs and excited at $260 \mathrm{~nm}$ (nano LED source). 


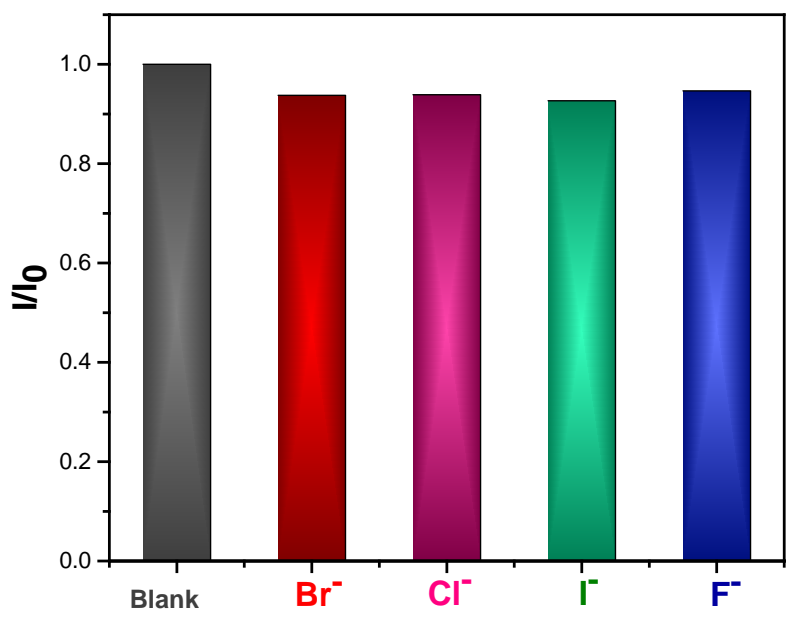

Figure S5. Photoluminescence intensity of three CDs in the absence and presence of anions.

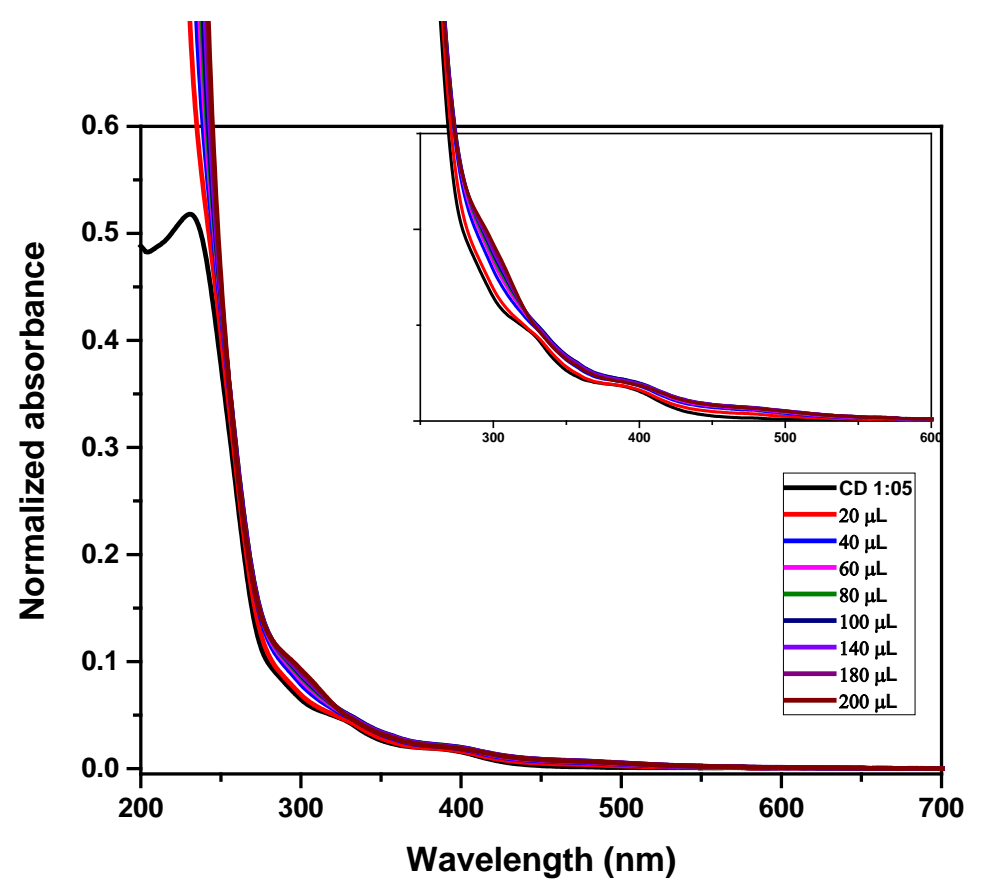

Figure S6. Absorption spectra of CD1:0.5 upon addition of different concentration of $\mathrm{Hg}^{2+}(3 \mathrm{mM})$. 

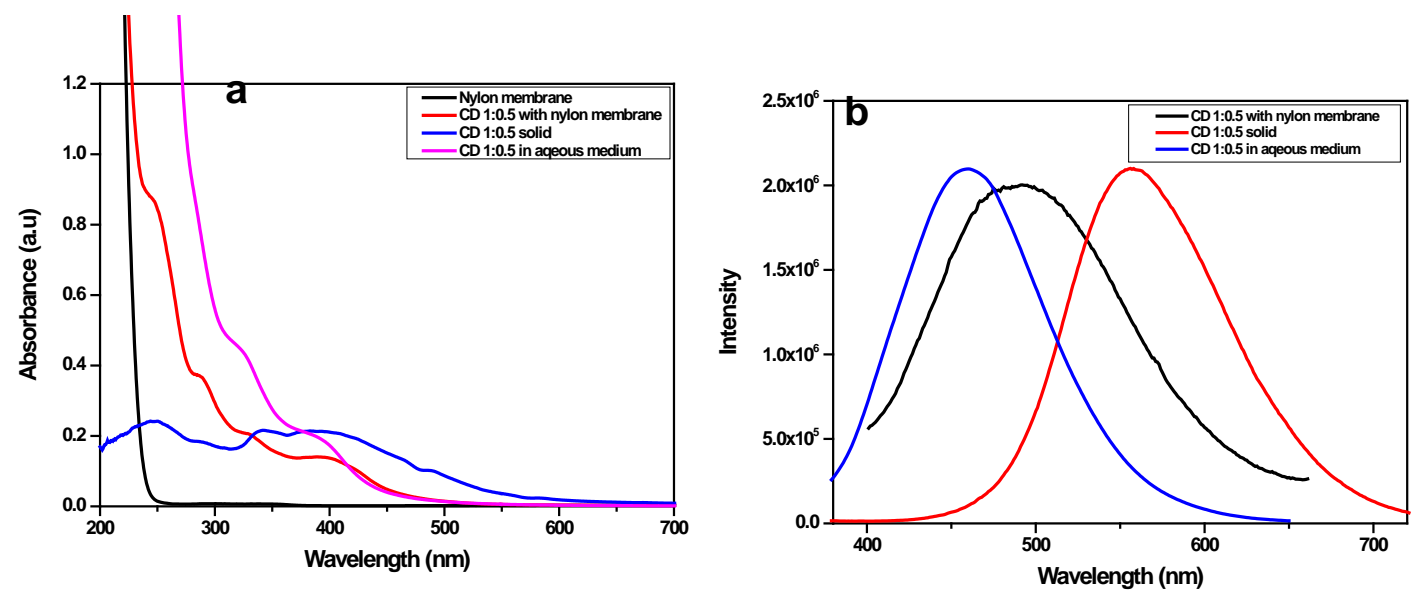

Figure S7. (a) DRS UV-Visible absorption spectra \& (b) Photoluminescence spectra of CDs (CD 1:0.5) fabricated nylon membrane with CDs in aqeous medium and solid state.
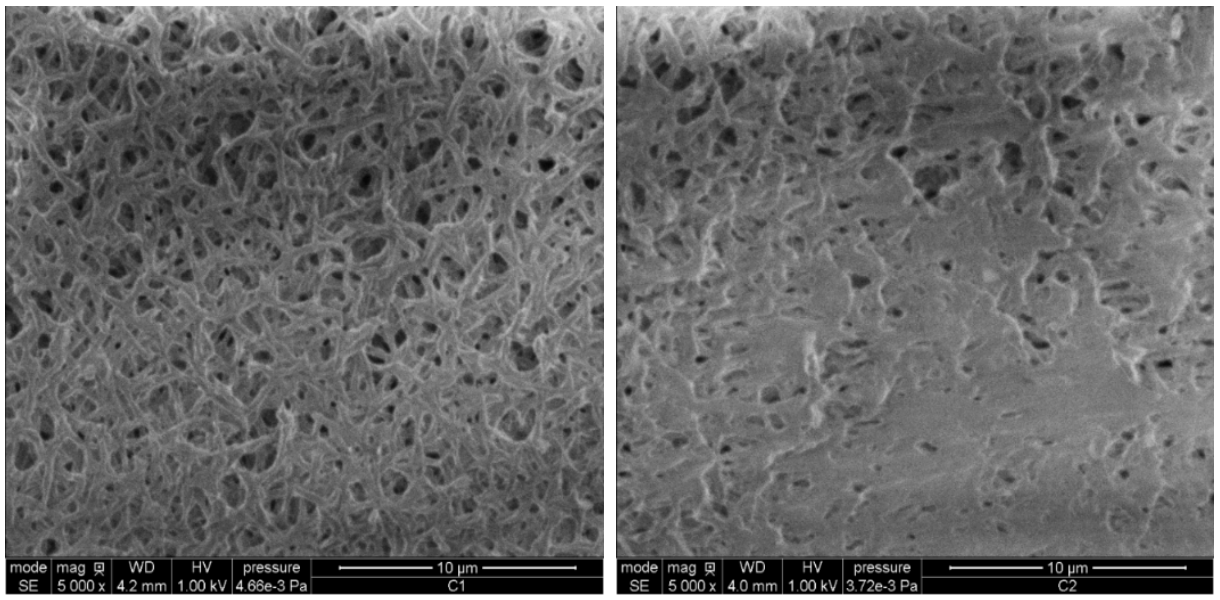

Figure S8. FESEM images of (a) pure nylon membrane and (b) high concentration of CD 1:0.5 fabricated nylon membrane 


\begin{tabular}{|l|l|l|l|l|}
\hline $\mathrm{Hg}^{2+}$ & Blank & $\mathrm{Cr}^{3+}$ & $\begin{array}{c}\text { Mixture of } \\
\mathrm{Fe}^{3+}, \mathrm{Cr}^{3+} \& \\
\mathrm{Hg}^{2+}\end{array}$ & $\mathrm{Fe}^{3+}$ \\
\hline
\end{tabular}

Figure S9. Metal ion interference study of CD1:0.5 fabricated strip probe under $365 \mathrm{~nm}$ UV lamp, after dropping of selective metal ions

Table S10. Summary table of elemental composition of CDs.

\begin{tabular}{cccc} 
& \multicolumn{3}{c}{ Weight \% } \\
CDs & C & N & O \\
CD 1:1 & 60.5 & 5.9 & 23.6 \\
CD 1:0.5 & 58.3 & 3.9 & 29.3 \\
CD 1:0.1 & 44.2 & 1.4 & 33.4 \\
\hline
\end{tabular}

